\title{
Review of mobile robots' obstacle avoidance, localization, motion planning, and wheels
}

\author{
Mustafa A. Mhawesh ${ }^{1}$, Zaid H. Al-Tameemi ${ }^{2}$, Omar Muhammed Neda ${ }^{3}$ \\ ${ }_{1,2}$ Al-Furat Al-Awsat Technical University, Iraq \\ ${ }^{3}$ Department of Engineering Affairs, Sunni Diwan Endowment, Iraq
}

\section{Article Info \\ Article history: \\ Received Feb 16, 2020 \\ Revised Apr 15, 2020 \\ Accepted Apr 29, 2020}

\section{Keywords:}

Mobile robots

Mobile robots wheels

Monte carlo localization

Motion planning

Obstacle avoidance robots

\begin{abstract}
The main objective of this research is to study the obstacle avoidance, monte carlo localization (MCL) method, motion planning in dynamic networks for mobile robots, and mobile robots wheels depending on the previous published researches. The researchers had done their experiments on different mobile robots and had validated them. This research helps the readers to learn how the robot changes its directions to prevent itself from collisions depending on three ultrasonic sensors. Also, they will learn the localization of the mobile robots depending on the recorded data from RHINO and MINERVA robots. In addition to learning the obstacle avoiding and the localization of mobile robots, the readers will learn new planning framework. Furthermore, they will get knowledge in types of mobile robots wheels.
\end{abstract}

Copyright $\odot 2020$ Institute of Advanced Engineering and Science. All rights reserved.

\section{Corresponding Author:}

Mustafa A. Mhawesh,

Al-Furat Al-Awsat Technical University, Iraq,

Kufa, Najaf, Iraq.

Email: mustafaazzam@atu.edu.iq

\section{INTRODUCTION}

The mobile robots are very useful in different fields, so the researchers are eager to work many researches on them. They are used in space exploration, military, medicine, toys, and in education. They are used underwater exploration, as well. Mobile robots have been created to have characteristics similar to humans or animals characteristics $[1,2]$.

The obstacles, the localization, and the motion planning without crash are the challenges that face the mobile robots. This research will discuss these challenges depending on the previous published researches. In addition to the challenges, the research will go through the types of wheels. The second section studies the obstacle avoiding robot by using three ultrasonic sensors. The third section explains method of Monte Carlo Localization, MCL to determine a mobile robot location regardless the starting location. The fourth section discusses the motion planning in dynamic networks. It is going to present the experiments to validate the approach. The section five states the mobile robots wheels.

One of the significant challenges that faces the mobile robots is the obstacles. Therefore, the researchers of [3] proposed an algorithm and named it "the bubble rebound algorithm". This algorithm deals with real time obstacle avoidance. The obstacles that are sensed in the region surrounded the robot are considered, so this region is called "sensitivity bubble".the researchers of [4] proposed an idea making the mobile robots discover and avoid the obstacles. The novelty was called Virtual Force Field. Another challenge that faces the mobile robots is the localization. The localization can be defined as determination of the robot location depending on the data that obtained from sensors [5-8].The researchers of [9] presented an idea to address the problem of localization for several robots. Their idea depended on polynomial calculations and got results by simulation. The algorithm of localization on robot operating system, ROS, is discussed in the research of [10]. The researchers analyzed the effects of particles to localize the robot position. From their experiments, 
more actual robot position can be obtained by using more particles. Furthermore, the authors of [11] enhanced the Monte Carlo Localization, MCL by using selective adaptive samples, SAMCL to address the issues of single and multi-robot localizations. Their proposal was achieved by using pre-caching technique, similar energy region, SER, and localization. The motion planning without crash is a challenge that faces the mobile robots, as well. The motion planning is the path between the initial position and the destination of mobile robots trip. Along their trip may contain known or unknown obstacles. How to determine free collision path is the research that Authors of [12] did. They presented an algorithm for mobile robot to plan a path in local environments with stationary and moving obstacles. The authors of [13] suggested a potential field method for motion planning of mobile robots in a dynamic environment where the target and the obstacles are moving. The researchers of [14] proposed a decentralized approach for multiple mobile robots motion planning without collisions. The best thing in the proposed algorithm is not caring to the shape and amount of obstacles. Their approach has the simplicity and effectiveness in realization the conflict free of multi mobile robots that operate in a common workplace. About the mobile robots wheels types, the authors of [15] proposed a new approach to one of the types which is the omnidirectional wheels. The omnidirectional wheels usually have three DOFs in motion, but the authors added one DOF to them. They added the steerable property to the omnidirectional wheels and named their approach an omnidirectional mobile robot with steerable omnidirectional wheels or OMR-SOW. Their proposal enhanced the continuously variable transmission, CVT action in which robot stability is assured without caring to how the wheels are arranged. Consequently, a great increasing happens in velocity ratio. A design was suggested to Mecanum wheel to enhance the efficiency of mobile robots [16]. The researchers of [17] designed a new universal omnidirectional wheels and named them continuous alternate wheels, CAW. They filled the gaps by inner rollers.

\section{OBSTACLE AVOIDING ROBOT}

The authors of [18] used three ultrasonic distance sensors to let the robot detect the obstacles and avoid them without any collisions. The researchers designed the robot to work on the ground. The obstacles detection is above the surface in real time, and path planning is not required. These sensors measure the distance to surrounding objects by emitting an ultrasonic pulse every $300 \mathrm{~ms}$ that echoes from the neighboring objects. The distance is calculated by using Arduino UNO depending on the constant speed of the sound $340 \mathrm{~m} / \mathrm{s}$ and the spent time between the input and echo. The robot detours its way when a sensor detects an obstacle within threshold distance. However, if all sensors detect obstacles within their range, the robot will move backward for $10 \mathrm{~ms}$. After that, the robot investigate which the way is better to move by helping from right and left sensors depending on the larger distance. The Figures 1, 2, and 3 show the front view of the used robot, algorithm for obstacle avoiding robot, and flowchart of hardware components of the used robot respectively [18].

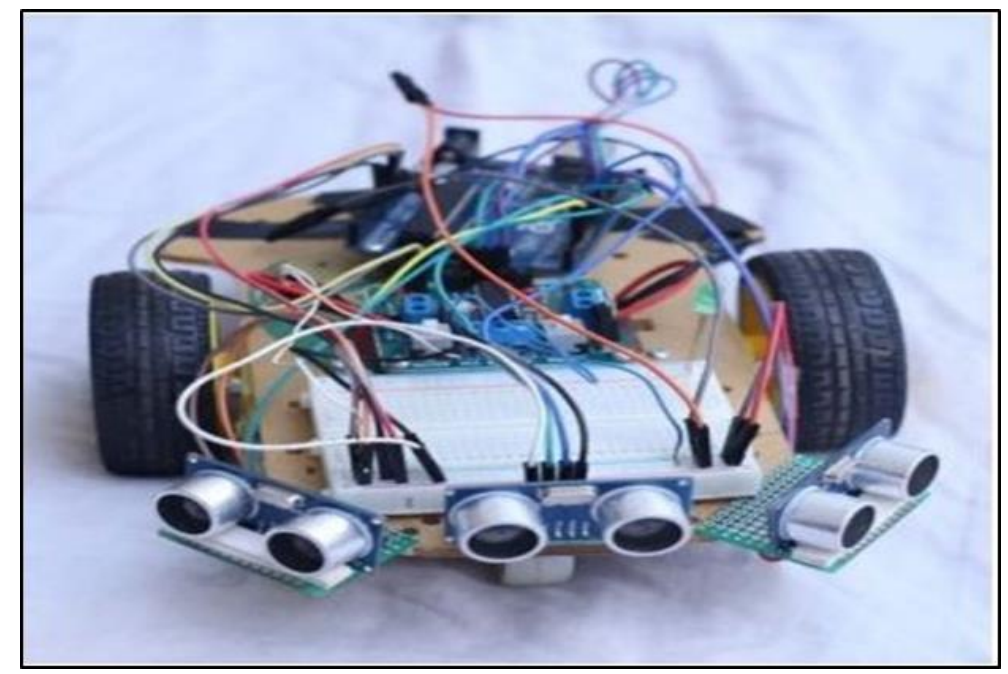

Figure 1. Front view of the robot [18] 


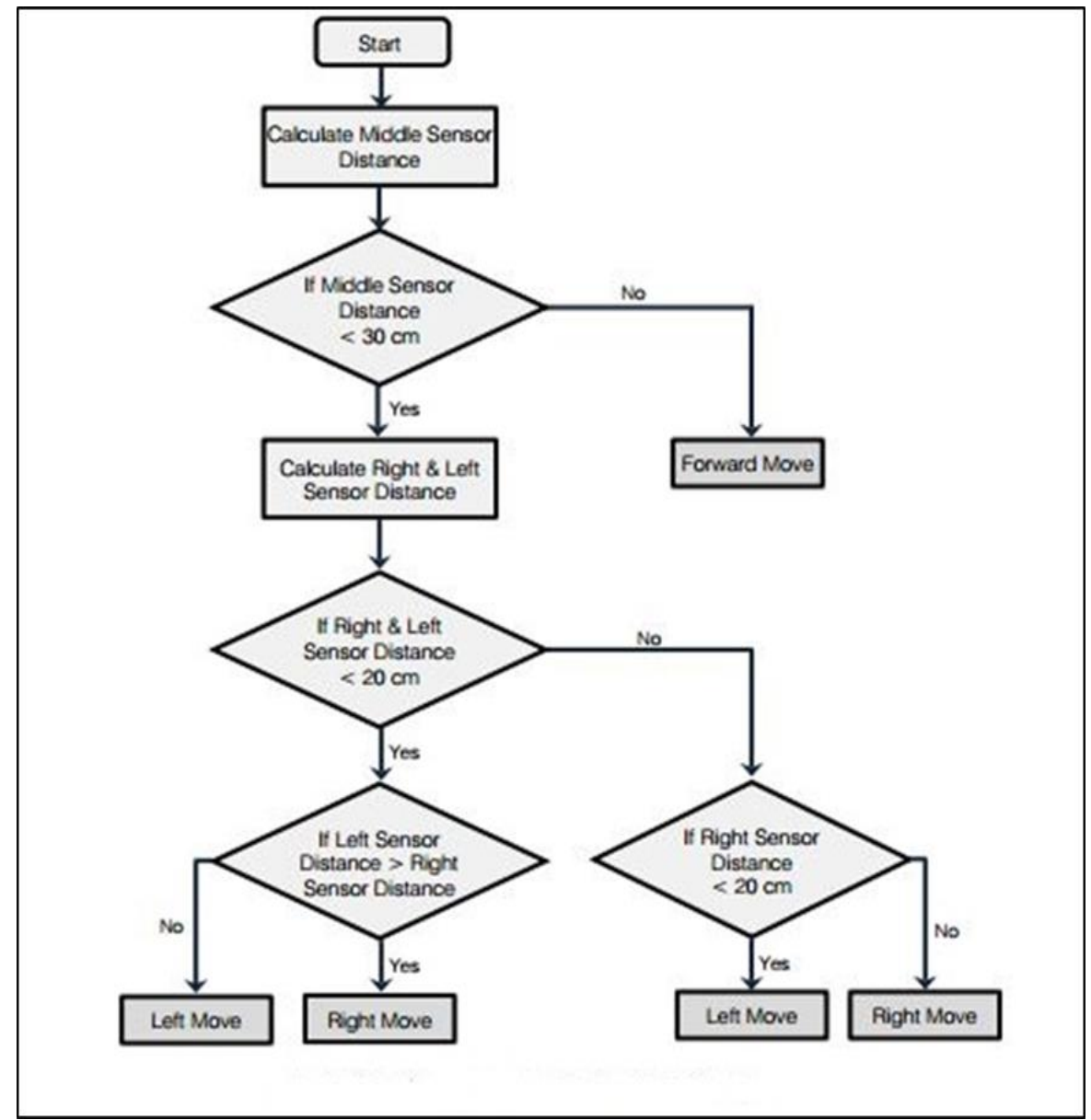

Figure 2. Algorithm for obstacle avoiding robot [18]

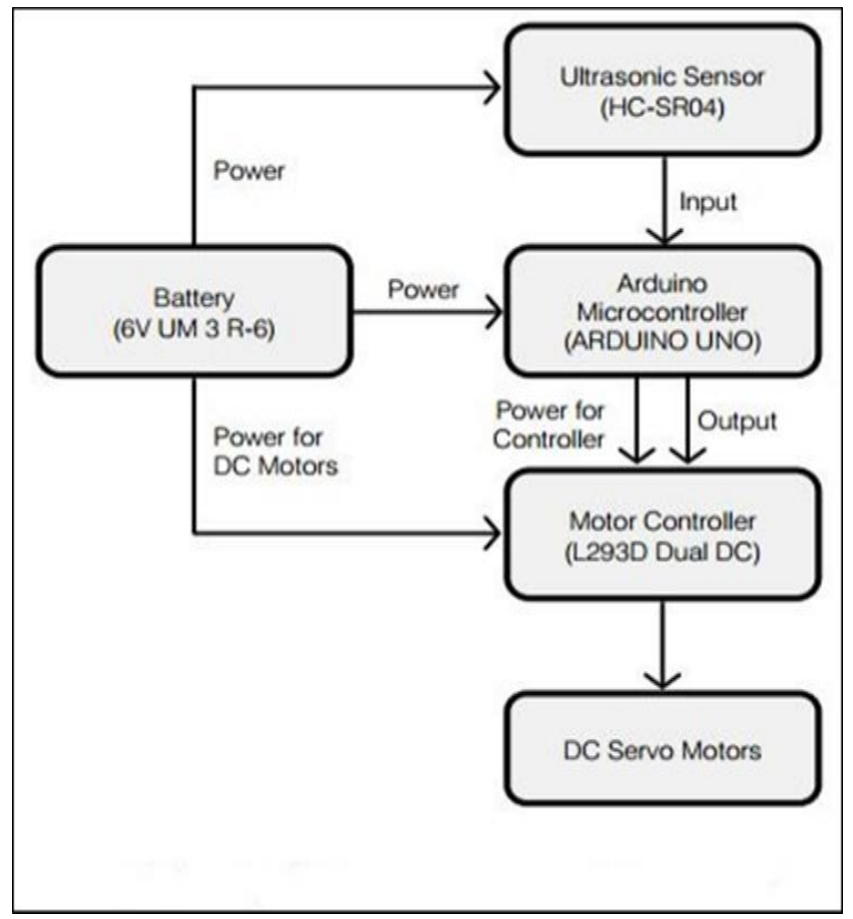

Figure 3. Flowchart of hardware components of the used robot [18] 


\section{MONTE CARLO LOCALIZATION FOR MOBILE ROBOTS}

The authors of [19] worked on the estimation of mobile robot position. They had used different robotic platforms in their office environment to test the Monte Carlo Localization, MCL. The MCL method can perform multi- modal probability distributions, and mobile robot localization can be performed without knowledge the starting location. They recorded the data from RHINO and MINERVA robots shown in the Figure 4 to do their experiments. They used sampling/importance resampling (SIR) process to prove their graph theoretically [19].

They ran their MCL-method on the recordings from RHINO, while they varied the amount of samples used to represent the density. A cell size plays a significant role to improve the accuracy as shown in the Figure 5 [19]. Furthermore, even though imprecise occupancy grid maps is used and when the robot moves very fast, the position of a robot for long time can be tracked by the MCL-method. The researchers did their experiment in Smithsonian's National Museum of American History. The recorded laser data from the robots are used, which the robots speed was up to $1.6 \mathrm{~m} / \mathrm{s}$ through the museum. There is no visitors in the museum during the test. The World Wide Web was used to control the robots. This run spent for seventy five minutes where the robot travels over 2200 meters, which the algorithm never once lost track. The area that the robot was run was 40 by 40 meters as shown in the Figure 6 . They used 5000 samples for the particular tracking experiment. Generally, the samples which are required for position tracking are far fewer than for global localization [19].

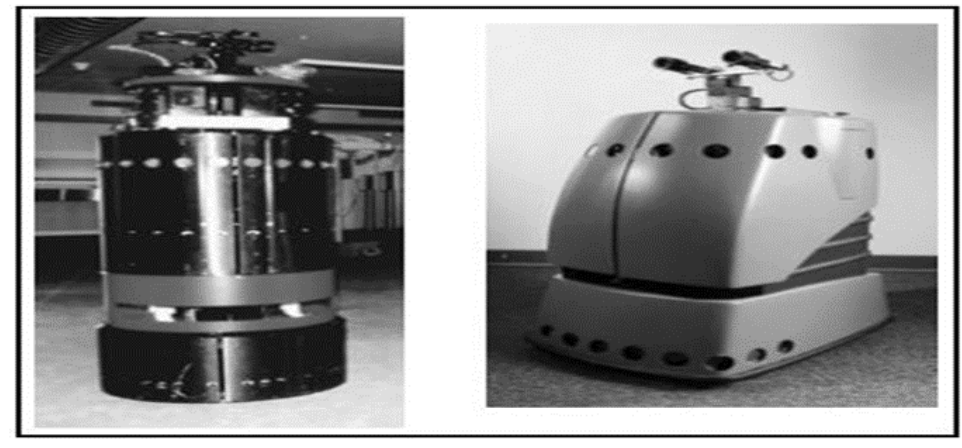

Figure 4. The robots RHINO (left) and MINERVA (right) [19]

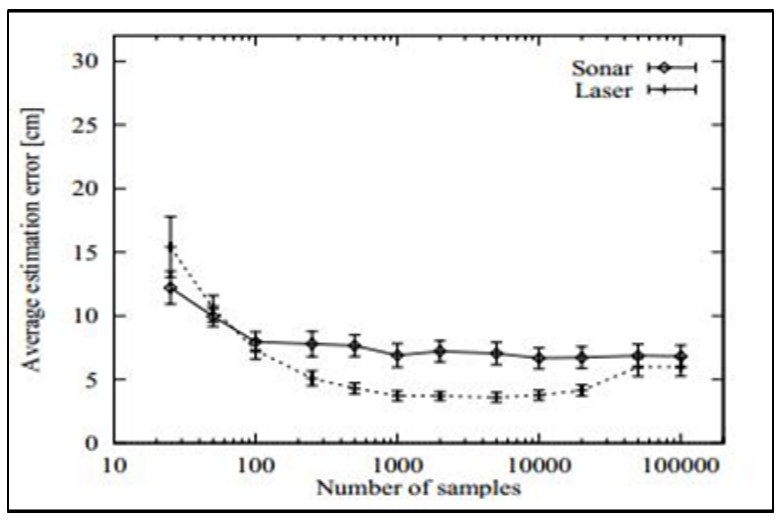

Figure 5. Log scale represents the accuracy of the method by using different amount of samples [19, 20]

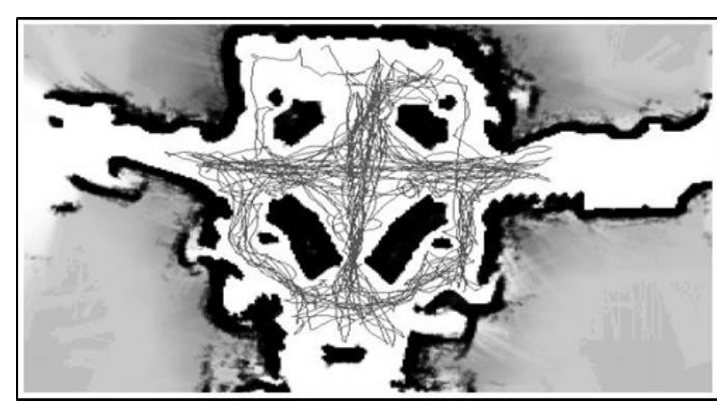

Figure 6. The robots worked area in the museum with a successful track of over 2000m [19, 21]

\section{MOBILE ROBOTS MOTION PLANNING}

A new planning framework is achieved in the research of $[22,23]$.The approach depends on dynamic robot networks that they can do three things.

a) Whenever communication and sensing capabilities permit, the networks can be formed dynamically.

b) The robots destinations and the global models in each network are being shared.

c) A fast-centralized motion planner is used to construct "on the fly" the trajectories coordination for the robots in each network. 


\subsection{Planning process in dynamic networks}

One of the following cases triggers the motion planning in a network:

a) Two robots from network enters the other's communication network.

b) An important variation in the world model happens. For example, a robot in a network detects a new obstacle.

c) A new destination is needed for a robot or more robots.

Each robot has information; it will share the information to its group in the network. After that, each robot runs a centralized motion planner to construct trajectories coordination. These coordinated trajectories will be for the robots in the network. Then the robots will execute the trajectory in the best plan. The planner is a single-query probabilistic-roadmap (PRM) planner [22].

\subsection{Test-platform}

\subsubsection{Micro-autonomous rovers test-platform}

There are several cylindrical robots with two driven wheels are used in the test. These robots are moving over surface of granite table, which its dimensions is $(3 \mathrm{~m} \times 2 \mathrm{~m})$. The table and the robots are the contents of the Micro-Autonomous RoverS (MARS) platform test [22].

\subsubsection{Sensors}

The states of all obstacles on the table are tracked by using an overhead vision system. The processor of this system computes the states and gives the information to every application that subscribes. Consequently, the information is going to be available to all robots. The obstacle states are filtered to make simulation for the limited detection range, which would happen when sensors are placed on robots [22].

\subsubsection{Network communication}

The computer-network architecture of the MARS test-platform is shown in the Figure 7. Whole the processing is achieved off board. For planning and controlling, each robot has two processors. The computers connection is LAN. The Real Time Innovation's network data delivery service (NDDS) software is used to communicate within the LAN. NDDS depends on a publish-subscribe architecture [22].

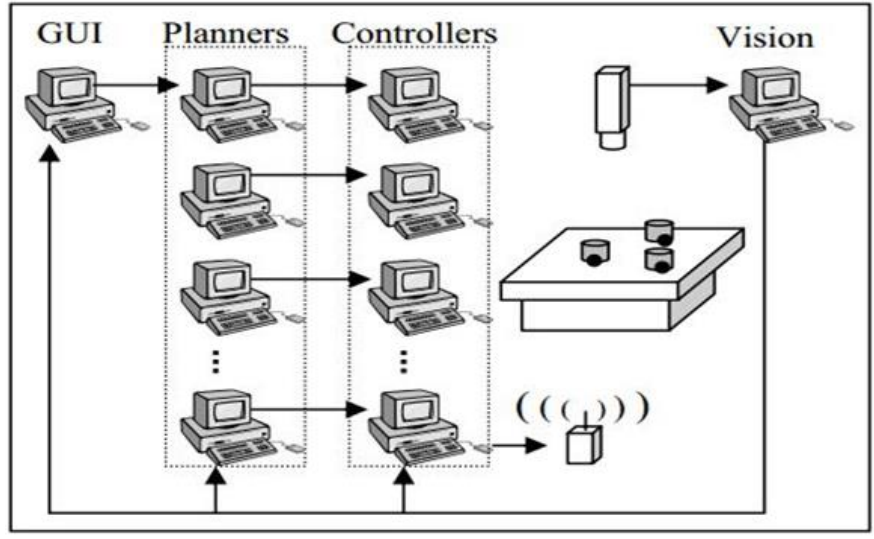

Figure 7. The Architecture of MARS test-platform [22]

\subsection{Experiments}

\subsubsection{Physical experiments}

Five real robots had been used to do the experiments where they located on the table, close to its bottom end. The ranges of sensors and the communication were limited to $75 \mathrm{~cm}$. There were two forms of networks on startup, one of them in the left and the other in the right. The robots tracks their trajectories to arrive their destinations at the end of the table. During the experiment, the average planning times was spent $9 \mathrm{~ms}$, and the robots planned an average 3.4 times. Figure 8 illustrates the example experiment on the MARS test platform. Five real robots and three obstacles are used. The right side of the figure is the physical hardware, and the left side represents the GUI screen-shots and corresponds the real components of the experiment. In GUI, small circles, large circles, cross-hairs, and lines reaching to the destinations depict robots, obstacles, destinations, and the trajectories respectively [22]. 


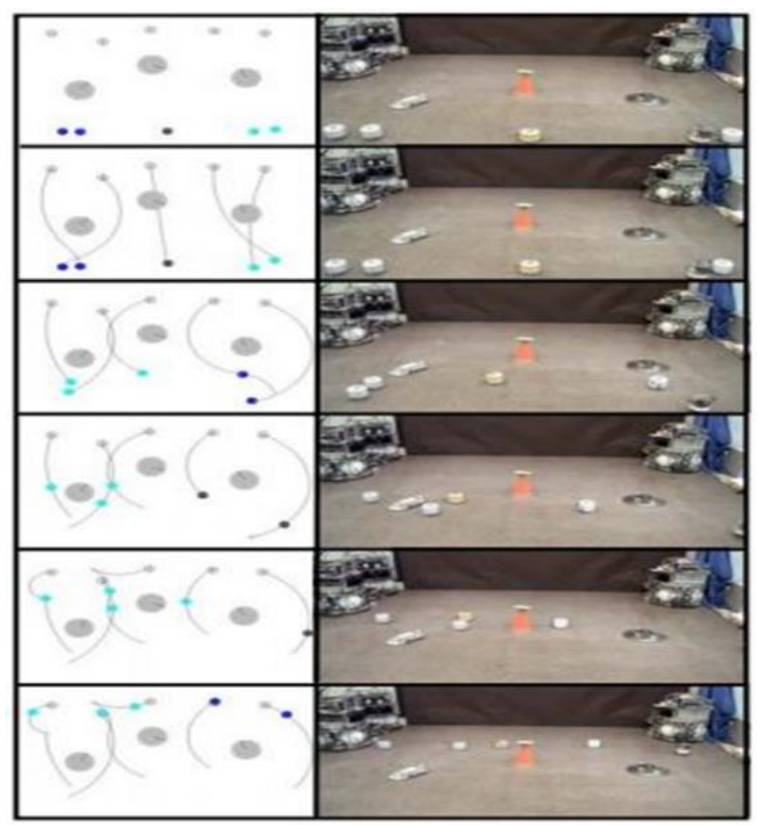

Figure 8. MARS test-platform consists of five robots and three obstacles [22]

\subsubsection{Simulations}

To make more complex experiments, twelve robots and twelve obstacles were used in $(4 \mathrm{~m} \times 6 \mathrm{~m})$ workspace. The twelve obstacles are six stationary and six moving robots. Both robots and obstacles had diameters $14 \mathrm{~cm}$. Four obstacles from the moving obstacles were moving to reach a network of robots as shown in the middle of the Figure 9. Likewise, two networks with two robots were put between a set of three obstacles and workplace borders. This procedure was executed twenty five times by using different initial random seeds. In spite of the hardness of the procedure, planner clearly shows fast planning times, an average of $15.8 \mathrm{~ms}$, while planning for up to five robots in a network. The authors of [9] did test with level of complexity and got simulation data by forming robots on average forty nine different networks during several minutes. The Simulation data for the test is shown in the Table 1.

Table1. Simulation data for the test scenario [22]

\begin{tabular}{ll} 
Average number of robots per plan & 2.12 \\
Average planning $(\mathrm{ms})$ & 17.3 \\
Average number of plans per robot per simulation & 5.07 \\
Average number of networks formed per simulation & 49.4 \\
\hline
\end{tabular}

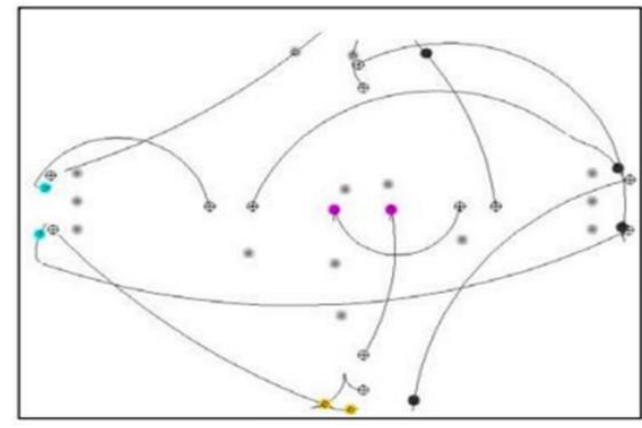

Figure 9. Screen-shot of the test scenario [22]

\section{MOBILE ROBOTS WHEELS}

There are four types of wheels that are used in robots: standard, orientable, ball, and omnidirectional wheels.

\subsection{Standard wheels}

They are called conventional wheels. They have two DOFs [24-26]. They have cylindrical shape. They are used to drive the robots forward and backward and to steer the robots right and left. The circumference of the wheels are surrounded by a traction material such as rubber or polyurethane to make a friction between the wheels and the ground [27]. The Figure10 shows this type of wheels. 

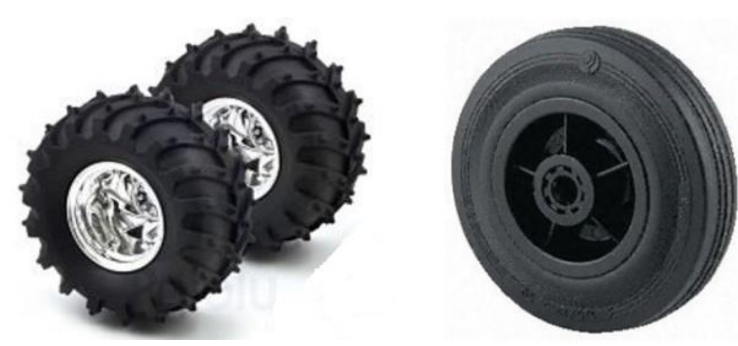

Figure 10. Standard wheels [26]

\subsection{Orientable wheels}

The orientable wheels are similar to the standard wheels, but they are placed to robots base by fixed or multi direction fork. The wheels are used for balance the robots not for driving them. This type of wheels can be classified to centered orientable wheels and off-centered orientable wheels depending on the vertical axle of the wheel. If the vertical axle passes in the wheel center, the orientable wheels are centered orientable wheels. However, if the vertical axle does not pass in the wheel center, the orientable wheels are off-centered orientable wheels [26]. The off-centered orientable wheels are also called castor wheels. The Figure 11 shows this type of wheels.
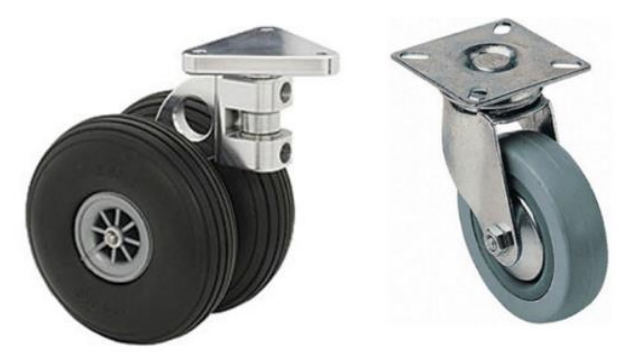

Figure 11. Orientable wheels [26]

\subsection{Ball wheels}

These wheels have spherical shape with three DOFs. They enable robots to maneuver [24, 25]. They are used for balance robots. Their balls are manufactured from metals or plastics. They are placed on the robots frame by holder [26]. The Figure 12 shows this type of wheels this type of wheels.
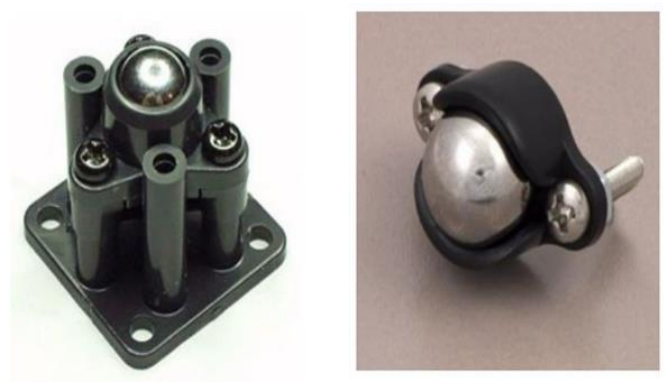

Figure 12. Ball wheels [26]

\subsection{Omnidirectional wheels}

These wheels are multidirectional wheels, which give the robots the ability to move in any direction. The omnidirectional wheels contain rollers on their circumferences. The rollers are made from composited rubber to be helpful in the friction [27]. This type of wheels has three DOFs. One of them represents the direction of the wheel orientation. The second of them represents the roller motion. The third represents 
the rotation slip on the point of touch between the wheel and the ground $[24,25]$. These wheels are used to drive and steer the robots [26]. They are called Swedish wheels because they are designed by Swedish engineer, Ilon, in Mecanum Company in 1973. If the rollers are placed on the circumference of the wheel with $45^{\circ}$ with respect to the base axis, the wheels are called Mecanum wheels. If they are aligned by $0^{\circ}$, the wheels are called universal wheels [15, 27-29]. The Figure 13 shows the omnidirectional wheels.
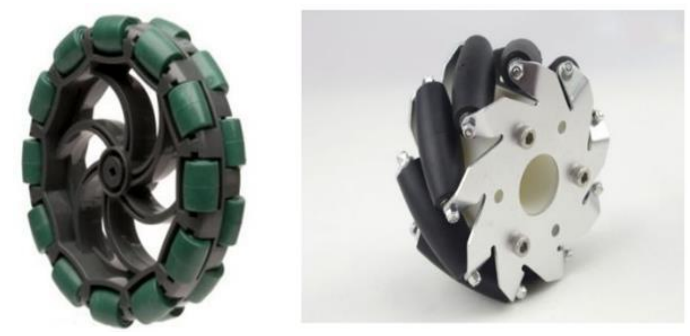

Figure 13. Omnidirectional wheels. The left is universal omnidirectional wheels and the right is mecanum wheels [27]

\section{CONCLUSION}

This research presents a detailed study about mobile robots. It is based on researches that are already published. The researchers proved their ideas by doing experiments. The researchers of obstacles avoidance robots achieved their idea by using three ultrasonic sensors. The researchers of the MCL achieved their idea by using recorded data from RHINO and MINERVA robots. The researchers of motion planning in dynamic networks by using MARS test-platform. Finally, each type of mobile robots wheels are explained and where they are used.

\section{REFERENCES}

[1] B. Crnokic, M. Grubisic and T. Volaric, "Different Applications of Mobile Robots in Education," International Journal on Integrating Technology in Education (IJITE), vol. 6, no. 3, pp. 15-28, 2017.

[2] B. Crnokić, S. Rezić and S. Pehar, "Comparision of Edge Detection Methods for Obstacles Detection in a Mobile Robot Environment," in 27th DAAAM International Symposium on Intelligent Manufacturing and Automation, pp. 0235-0244, 2016.

[3] I. Susnea, V. Minzu and. G. Vasiliu, "Simple, Real-Time Obstacle Avoidance Algorithm for Mobile Robots," 8th WSEAS International Conference on Computational Intelligence man-machine Systems and Cybernetics (CIMMACS'09), pp. 24-29, 2009.

[4] J. Borenstein and Y. Koren, "Real-time Obstacle Avoidance for Fast Mobile Robots," in IEEE Transactions on Systems, Man, and Cybernetics, vol. 19, no. 5, pp. 1179-1187, 1989.

[5] D. Fox, W. Burgard and S. Thrun, "Active Markov localization for mobile robots," Robotics and Autonomous Systems, vol. 25, no. 3-4, pp. 195-207, 1998.

[6] W. Burgard, D. Fox and S. Thrun, "Active mobile robot localization by entropy minimization," in Proceedings of Second EUROMICRO workshop on Advanced Mobile Robots, Brescia, Italy, pp. 155-162, 1997.

[7] D. Fox, W. Burgard and S. Thrun, "Markov Localization for Mobile Robots in Dynamic Environments," Journal of Artificial Intelligence Research, vol. 11, pp. 391-427, 1999.

[8] S. Thrun, D. Fox, W. Burgard and F. Dellaert, "Robust Monte Carlo Localization for Mobile Robots," Artificial Intelligence, vol. 128, no. 1-2, pp. 99-141, 2001.

[9] J. R. Spletzer and C. J. Taylor, "A Bounded Uncertainty Approach to Multi-Robot Localization," Proceedings 2003 IEEE/RSJ International Conference on Intelligent Robots and Systems (IROS 2003) (Cat. No.03CH37453), Las Vegas, NV, USA, vol. 2, pp. 1258-1265, 2003.

[10] F. R. Q. Aini, A. N. Jati and U. Sunarya, “A study of Monte Carlo localization on robot operating system," 2016 International Conference on Information Technology Systems and Innovation (ICITSI), Bandung, 2016.

[11] L. Zhang, R. Zapata and P. L'epinay, "Self-Adaptive Monte Carlo for Single-Robot and Multi-Robot Localization," 2009 IEEE International Conference on Automation and Logistics, Shenyang, pp. 1927-1933, 2009.

[12] S. K. Das, A. K. Dutta and S. K. Debnath, "Operative Critical Point Bug algorithm-local path planning of mobile robot avoiding obstacles," Indonesian Journal of Electrical Engineering and Computer Science (IJEECS), vol. 18, no. 3, pp. 1646-1656, 2020.

[13] S. S. GE and Y. J. CUI, "Dynamic Motion Planning for Mobile Robots Using Potential Field Method," Autonomous Robots, vol. 13, pp. 207-222, 2002.

[14] K. Azarm and G. Schmidt, "Conflict-Free Motion of Multiple Mobile Robots Based on Decentralized Motion Planning and Negotiation," Proceedings of International Conference on Robotics and Automation, Albuquerque, NM, USA, vol. 4, pp. 3526-3533, 1997. 
[15] J. Song and K. Byun, "Design and Control of a Four-Wheeled Omnidirectional Mobile Robot with Steerable Omnidirectional Wheels," Journal of Robotic Systems, vol. 21, no. 4, pp. 193-208, 2004.

[16] O. Diegel, A. Badve, G. Bright, j. Potgieter and S. Tlale, "Improved Mecanum wheel Design for Omni-Directional Robots," in Australasian Conference on Robotics and Automation, Auckland, pp. 117-121, 2002.

[17] K.-S. Byun, S.-J. Kim and J.-B. Song, "Design of Continuous Alternate Wheels for Omnidirectional Mobile Robots," in Proceedings 2001 ICRA. IEEE International Conference on Robotics and Automation (Cat. No.01CH37164), Seoul, South Korea, vol. 1, pp. 767-772, 2001.

[18] F. Tabassum, S. Lopa, M. M. Tarek and B. J. Ferdosi, "Obstacle Avoiding Robot," Global Journal of Researches in Engineering, vol. 17, no. 1, pp. 18-24, 2017.

[19] F. Dellaert, D. Fox, W. Burgard and S. Thrun, "Monte Carlo Localization for Mobile Robots," Proceedings 1999 IEEE International Conference on Robotics and Automation (Cat. No.99CH36288C), Detroit, MI, USA, vol. 2, pp. 1322-1328, 1999.

[20] D. Fox, W. Burgard, F. Dellaert and S. Thrun, "Monte Carlo localization: Efficient position estimation for mobile robots," in Proceedings of the Sixteenth National Conference on Artificial Intelligence and Eleventh Conference on Innovative Applications of Artificial Intelligence, Orlando, 1999.

[21] S. Thrun, D. Fox and W. Burgard, "Monte Carlo Localization With Mixture Proposal Distribution," in Proceedings of the AAAI National Conference on Artificial Intelligence, pp. 1-7, 2002.

[22] C. M. Clark, S. M. Rock and J. Latombe, "Motion planning for multiple mobile robots using dynamic networks," 2003 IEEE International Conference on Robotics and Automation (Cat. No.03CH37422), Taipei, Taiwan, vol. 3, pp. 4222-4227, 2003.

[23] C. Clark, S. Rock and J. Latombe, "Dynamic Networks for Motion Planning in Multi-Robot Space Systems," Proceedings of the 7th International Sympsium on Artificial Intelligence, Robotics, and Automation in Space, 2003.

[24] P. F. Muir and C. P. Neuman, "Kinematic modeling of wheeled mobile robots," Carnegie-Mellon Unhrenity, Pittsburgh, Pennsylvania, 1986.

[25] P. F. M. a. C. P. Neuman, "Kinematic modeling of wheeled mobile robots," Journal of Robotic Systems, vol. 4, no. 2, pp. 281-340, 1987.

[26] C. D. George, "Overview of Wheels Used in Robotics," Intorobotics, 2013.

[27] J. J. Parmar and C. V. Savant, "Selection of Wheels in Robotics," International Journal of Scientific and Engineering Research, vol. 5, no. 10, pp. 339-343, 2014.

[28] M. O. Tătar, C. laudiu Cirebea and D. Mândru, "Structures of the omnidirectional robots with swedish wheels," Solid State Phenomena, vol. 198, pp. 132-137, 2013.

[29] G. Indiveri, "Swedish wheeled omnidirectional mobile robots: Kinematics analysis and control," in IEEE Transactions on Robotics, vol. 25, no. 1, pp. 164-171, 2009.

\section{BIOGRAPHIES OF AUTHORS}

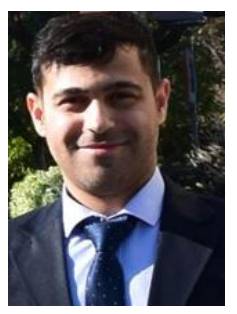

Mustafa A. Mhawesh earned his master's degree in Electrical Engineering from California State University, Fullerton, USA in 2017. He earned the B.Sc degree from AL-Furat Al-Awsat Technical University, Iraq 2011. He is working as assistant lecturer in Al-Furat Al-Awsat Technical University, Iraq. His research interests are robotics and control systems.

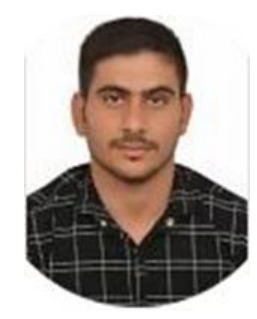

Zaid H. Al-tameemi received his master's degree in the field of electrical power engineering from the University of South Australia, Australia, 2017. He received the B.Sc degree from ALFurat Al-Awsat Technical University, Iraq 2010. He is working as assistant lecturer in the Technical College/al-Mussaib and his interests are renewable energy technologies and advanced machines.

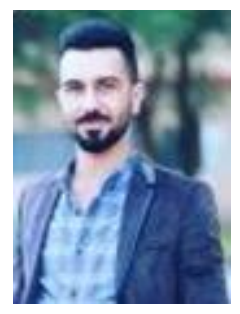

Omar Muhammed Neda received his master's degree in the field of electrical power engineering from Middle Technical University, Iraq, 2018. He received the B.Sc degree from AL-Furat Al-Awsat Technical University, Iraq, 2010. He is interested in Optimization Techniques, Electrical Machines, Power Systems Renewable Energy, and Artificial Intelligence. He is working as an assistant lecturer in Middle Technical University. 